\title{
VALIDATION PROCEDURE IN THE MODIFIED AMBIGUITY FUNCTION APPROACH
}

\author{
Sławomir CELLMER
}

Institute of Geodesy, University of Warmia and Mazury in Olsztyn, Poland

*Corresponding author's e-mail: slawomir.cellmer@gmail.com

\begin{tabular}{l} 
ARTICLE INFO \\
\hline Article history: \\
Received 10 January 2015 \\
Accepted 16 April 2015 \\
Available online 12 May 2015 \\
\hline
\end{tabular}

Keywords:

GNSS data processing

MAFA method

Validation methods

\begin{abstract}
Precise coordinates of control points, obtained from GNSS data processing can be utilized in geodynamic research. Periodic surveys allow for determination of displacements. They form a data set for geodynamic interpretation. Of particular importance is permanent monitoring of control points. This mode of measurement requires advanced methods of GNSS data processing. One such method is the Modified Ambiguity Function Approach (MAFA). So far many tests of this method have been performed and the results show it is efficient. It is even possible to obtain a good solution based on GNSS data from a single epoch. In this article, three validation procedures for the MAFA method are proposed. They are based on different principles than the validation methods in classic the approach of precise positioning, in which test statistics are formed from the quadratic forms of residuals associated with the most likely set of integer ambiguities and the second most likely set of integer ambiguities. In the MAFA method the proposed procedures are based on defining the confidence region of the float solution and then testing whether the final solution is included in this region. To test the new validation procedures an experiment was designed and performed. Single epoch solutions for some baselines have been analysed and the results of this research presented in this paper. Finally, some conclusions were drawn based on this analysis.
\end{abstract}

\section{INTRODUCTION}

An integral part of survey data processing is results validation. This is particularly important for precise measurements that provide data on deformation analysis, in geodynamic studies. In classic measurements, statistical tests often are applied for detecting outliers in a data set or for the examination of the significance of the displacement (Caspary, 2000; Nowel, 2015a, 2015b). Contemporary geodynamic research often utilizes GNSS as a tool for precise positioning in mountainous areas (Hefty, 2007; Kontny et al., 2006; Lidberg, 2010; Schenk et al., 2010; Wielgosz et al., 2011). The problem of the validation of the results is particularly important in precise, satellite positioning, in which the carrier phase data is processed. It is well known, that in the classic approach, e.g. in the LAMBDA method, the whole process of precise GNSS positioning includes three steps: float solution, integer ambiguity resolution (together with validation procedures) and fixed solution (Teunissen, 1995). Ambiguity validation has been a challenge for many years, and is still regarded as an open problem (Verhagen, 2004). Most validation procedures are based on testing the quadratic form of the least-squares residual for different combinations of ambiguities. Over the past few years, various ambiguity validation methods have been proposed, e.g. the $F$-ratio test (Frei and Beutler, 1990; Euler and Landau, 1992; Abidin, 1993), $R$-ratio test (Euler and Schaffrin, 1991; Leick, 2004; Teunissen and Verhagen, 2009), difference test
(Tiberius and Jon, 1995), projector test (Wang et al., 1998; Han, 1997), ellipsoidal integer aperture (EIA) estimator (Teunissen, 2003, 2005) and penalized integer aperture (PIA) estimator (Teunissen, 2004). A lot of research has been done on analysing statistical and probabilistic aspects of the integer ambiguity estimators (Teunissen, 1998a,b, 1999, 2002; Xu, 2006). Recently, comparative studies of different ambiguity validation methods have been performed (Wang et al., 2000; Li and Wang, 2012; Teunissen, 2013). In the case of real time deformation monitoring it is necessary to apply a precise, positioning technique, that gives results instantaneously. One very promising method of real time, precise positioning is the Modified Ambiguity Function Approach (MAFA). This method of carrier phase processing is based on least squares adjustment with condition equations in the functional model of the adjustment problem (Cellmer et al., 2010; Cellmer 2011a, 2011b). The ambiguities are not explicitly solved in this approach. However their integer nature is preserved in the final solution, due to condition equations. The functional model for the carrier phase adjustment is relatively weak. Therefore different techniques for improving the efficiency of the MAFA method have been proposed (Cellmer, 2011a, 2011b, 2013). Three of them are the most important: cascade adjustment, integer de-correlation and search procedure. These procedures allow obtaining the correct solution, even if the a priori position is several metres away from the actual one. Until now, many 
tests of single-epoch positioning, using the MAFA method have been carried out (Cellmer, 2013). The results of the tests are very promising, and preliminary studies concerning the necessary condition for the MAFA method have been carried out. The results of these studies are presented in (Cellmer, 2012a). However, until now, no reliable validation technique has yet been developed. In this paper, three validation techniques are proposed. The foundations of these techniques are different from the above-mentioned validation procedures, which are implemented in classical methods of GNSS data processing. Each of them is based on forming a confidence region and then testing whether the final solution is inside it or not. The idea for this approach was inspired by the method of testing the significance of displacement in deformation measurement theory (Caspary et al., 1990; Chen, 1983).

The next section presents the foundations of the MAFA method. The techniques improving its efficiency are also presented. The third section contains a description of the proposed validation techniques. In the fourth section, the experiment is described. Thereafter, the results of the tests are analyzed and presented in a graphical form. Some conclusions have been drawn from this analysis and are presented in the last section.

\section{MAFA METHOD}

The MAFA method has been widely described in many articles (Cellmer et al., 2010, 2011a, 2011b, 2012a, 2013).

The following model for a double differenced (DD) carrier phase observable is assumed, (HofmannWellenhof et al., 2008; Leick, 2004; Teunissen and Kleusberg, 1998):

$\Phi+v=\frac{1}{\lambda} \rho\left(\boldsymbol{x}_{\boldsymbol{c}}\right)+a$

where:

$$
\begin{aligned}
& \Phi-\text { DD carrier phase observable (in cycles) } \\
& \lambda-\text { signal wavelength } \\
& v-\text { residual (measurement noise) } \\
& \boldsymbol{x}_{\boldsymbol{c}}-\text { receiver geocentric radius vector } \\
& \rho\left(\boldsymbol{x}_{\boldsymbol{c}}\right) \text { - DD geometrical range } \\
& a-\text { integer number of cycles (DD initial } \\
& \quad \text { ambiguity) }
\end{aligned}
$$

Taking into account the integer nature of the ambiguity parameter $a$ and assuming that the residual values are much lower than half a cycle, the linearized general formula of the residual equations can then be shown in the following form (Cellmer et al., 2010):

$\nu=\frac{1}{\lambda} \boldsymbol{A x}+\boldsymbol{\delta}$

with:

$\boldsymbol{\delta}=\operatorname{round}\left(\boldsymbol{\Phi}-\frac{1}{\lambda} \boldsymbol{\rho}_{0}\right)-\left(\boldsymbol{\Phi}-\frac{1}{\lambda} \boldsymbol{\rho}_{0}\right)$

where:

$$
\boldsymbol{v} \text { - residual vector }(n \times 1),
$$

$\boldsymbol{x}$ - parameter vector (increments to a priori coordinates vector $\left.\boldsymbol{x}_{\boldsymbol{0}}\right)$,

$\boldsymbol{A}$ - design matrix $(n \times 3)$,

$\delta$ - vector of misclosures $(n \times 1)$,

$\boldsymbol{\rho}_{0}-$ DD geometric distance vector computed using a priori position and satellite coordinates.

The LS solution of (2) can be computed from:

$\boldsymbol{x}=-\lambda\left(\boldsymbol{A}^{\boldsymbol{T}} \boldsymbol{P} \boldsymbol{A}\right)^{-1} \boldsymbol{A}^{\boldsymbol{T} P}$

with $\boldsymbol{P}$ standing for the weight matrix. If no elevation dependent factors to weighting are applied, the weight matrix of DD carrier phase observations is formed as an inverse of the following covariance matrix:

$\boldsymbol{Q}=\sigma_{0}^{2}\left[\begin{array}{cccc}4 & 2 & \cdots & 2 \\ 2 & 4 & \cdots & 2 \\ \vdots & \vdots & \ddots & 2 \\ 2 & 2 & 2 & 4\end{array}\right]$

where $\sigma_{0}$ is a standard deviation of a carrier phase observation.

On the current state of development, decorrelation (Cellmer, 2011a, 2011b) and search (Cellmer, 2013) procedures are mandatory part of the MAFA method. If there is poor approximation of the a priori position, the cascade adjustment is applied (Cellmer et al., 2010). In the case of single-epoch positioning, the following formula for approximation of the ambiguity covariance matrix is used as a base for the de-correlation procedure:

$Q_{a}=\left[P-k P A\left(A^{T} P A\right)^{-1} A^{T} P\right]^{-1}$

where $k$-factor with value from the range $(0 ; 1)$, guarantees that the expression in the brackets is a positive definite matrix. This factor simulates the involvement of an additional group of observations in the positioning problem. An interpretation of coefficient $k$, has been presented in detail in (Cellmer, 2012b ).

The de-correlation procedure is necessary, because the DD ambiguities $\boldsymbol{a}$ are usually strongly correlated. Hence, fixing one value of ambiguity through rounding the first term in (4) to the nearest integer, has an impact on the rest of the ambiguities. Therefore, the correlation between ambiguities should be taken into account. An alternative way of solving this problem is to transform the observation equations into their equivalent form but with de-correlated ambiguities. This can be done using integer decorrelation $\boldsymbol{Z}$ matrix (Teunissen, 1995; Liu et al., 1999):

$Q_{a z}=Z Q_{a} Z^{T}$

where:

$\boldsymbol{Z}$ - integer de-correlation matrix

$\boldsymbol{Q}_{\boldsymbol{a}}$ - ambiguity covariance matrix

$\boldsymbol{Q}_{a z}-$ diagonal transformed ambiguity covariance matrix. 
By multiplying Equation (1) with $\boldsymbol{Z}$, one can obtain a new equation with a new integer ambiguity vector $\boldsymbol{a}_{z}$ :

$\Phi_{\mathrm{Z}}+\boldsymbol{v}_{Z}=\frac{1}{\lambda} \rho_{\mathrm{Z}}\left(\boldsymbol{x}_{C}\right)+\boldsymbol{a}_{\mathrm{Z}}$

In this way Equation (8) replaces Equation (1). The de-correlation procedure increases the probability of obtaining the correct solution. There are many various methods of finding the $\boldsymbol{Z}$ matrix (Hassibi and Boyd, 1998; Liu et al., 1999; Xu, 2001). In order to find the $\boldsymbol{Z}$ matrix, the ambiguity covariance matrix $\boldsymbol{Q}_{\boldsymbol{a}}$ is required. In the case of single-epoch precise positioning, this matrix is evaluated using (6).

If the a priori position is poor, then the vector of misclosures from (2) takes the following form:

$$
\boldsymbol{\delta}_{\mathrm{e}}=\operatorname{round}\left(\boldsymbol{\Phi}-\frac{1}{\lambda} \boldsymbol{\rho}_{0}\right)-\left(\boldsymbol{\Phi}-\frac{1}{\lambda} \boldsymbol{\rho}_{0}\right)+\boldsymbol{a}_{\mathrm{e}}
$$

Due to the integer values of the vector $\boldsymbol{a}_{\mathrm{e}}$ the search procedure is necessary. The search procedure will consist of testing the values of the objective function:

$\Psi=v^{T} P v$

for different vectors $\boldsymbol{a}_{\mathrm{e}}$. In order to reduce the search region, Cellmer has proposed, that vector $\boldsymbol{a}_{\mathrm{e}}$, consists only of the values $-1,0$ and 1 (Cellmer, 2013). Thus, the search procedure is based on the misclosure vector modifications, followed by a test of the resulting objective function values. If the procedures of decorrelation and searching are applied then the MAFA method can be used for single-epoch precise positioning. The fundamental problem in such a case is the validation of the solutions. The procedures of validation are proposed in the next section.

\section{VALIDATION PROCEDURES}

In classic methods of precise positioning, validation procedures are based on testing different candidates of integer ambiguities. Test statistics are formed from the quadratic forms of residuals associated with the most likely set of integer ambiguities and the second most likely set of integer ambiguities. As was mentioned in the Introduction, different statistical tests are applied in these methods. All of them are based on testing sets of candidates of integer ambiguities. The validation methods in the MAFA method are based on a different principle. Three procedures of validation are proposed here. All of them are based on determination confidence region for solution and then testing whether obtained solution fall into it or not. In the first two methods confidence region is determined in coordinate domain and in the last method in ambiguity domain. Below are presented foundations of these three procedures.

\section{PROCEDURE 1 (F-TEST)}

First test, based on F-distribution, examines a significance of position displacement in a coordinate domain. Two $\chi^{2}$-distributed statistics are formed:

$\mathrm{s}_{1}=d \boldsymbol{x}^{T} \boldsymbol{Q}_{\mathrm{xf}}^{-1} d x \sim \chi_{3}^{2}$

$\mathrm{s}_{2}=\boldsymbol{v}_{\mathrm{c}}^{\mathrm{T}} \boldsymbol{P}_{\mathrm{c}} \boldsymbol{v}_{\mathrm{c}} \sim \chi_{\mathrm{n}-\mathrm{m}}^{2}$

where:

$\boldsymbol{d} \boldsymbol{x}$ - components of distance between position of fixed solution and position of float solution

$\boldsymbol{Q}_{\mathrm{xf}}$ - cofactor matrix of position coordinates of float solution

$\boldsymbol{v}_{\mathrm{c}}$ - residuals vector of float solution

$\boldsymbol{P}_{\mathrm{c}}$ - weight matrix of float solution data set

$\mathrm{n}$ - number of observations in float solution

$\mathrm{m}$ - number of parameters in float solution

Subscript in $\chi^{2}$ - statistics denotes the number of degrees of freedom.

Cofactor matrix $\boldsymbol{Q}_{\mathrm{xf}}$ is computed as:

$\boldsymbol{Q}_{\mathrm{xf}}=\left(\boldsymbol{A}_{\mathrm{c}}^{\mathrm{T}} \boldsymbol{P}_{\mathrm{c}} \boldsymbol{A}_{\mathrm{c}}\right)^{-1}$

with $\boldsymbol{A}_{\mathrm{c}}$ as a float solution model matrix.

If $\mathrm{s}_{1}$ and $\mathrm{s}_{2}$ are both $\chi^{2}$-distributed random variables with 3 and $n-m$ degrees of freedom respectively, then the statistic $\mathrm{s}_{3}$ below is $F$ distributed:

$\mathrm{S}_{3}=\frac{\frac{1}{3} \mathrm{~S}_{1}}{\frac{1}{\mathrm{n}-\mathrm{m}} \mathrm{S}_{2}} \sim \mathrm{F}_{3, \mathrm{n}-\mathrm{m}}$

Hence, critical value $\gamma_{0}$ can be determined for degrees of freedom 3 and $n-m$ and for assumed confidence level $\mathrm{p}_{0}$ :

$\mathrm{P}\left(\mathrm{F}_{3, \mathrm{n}-\mathrm{m}}<\gamma_{0}\right)=\mathrm{p}_{0}$

The above equation is a base for the test:

f $\frac{s_{3}}{\gamma_{0}}>1$ then solution is rejected.

Otherwise it can be accepted.

\section{PROCEDURE 2 (( $\left.\chi^{2}-T E S T\right)$}

In some cases there can be no information about $\mathrm{S}_{2}$ statistic (12). In this case only one statistic is formed:

$\mathrm{s}_{4}=d \boldsymbol{x}^{T} \boldsymbol{C}_{\mathrm{xf}}^{-1} d x \sim \chi_{3}^{2}$

Hence, on the basis of the formula:

$\mathrm{P}\left(\chi_{3}^{2}>\gamma_{0}\right)=1-\mathrm{p}_{0}$

with assumed confidence level $\mathrm{p}_{0}$, the following test can be formulated:

$\mathrm{f} \frac{\mathrm{s}_{4}}{\gamma_{0}}>1$ then solution is rejected.

Otherwise it can be accepted. 

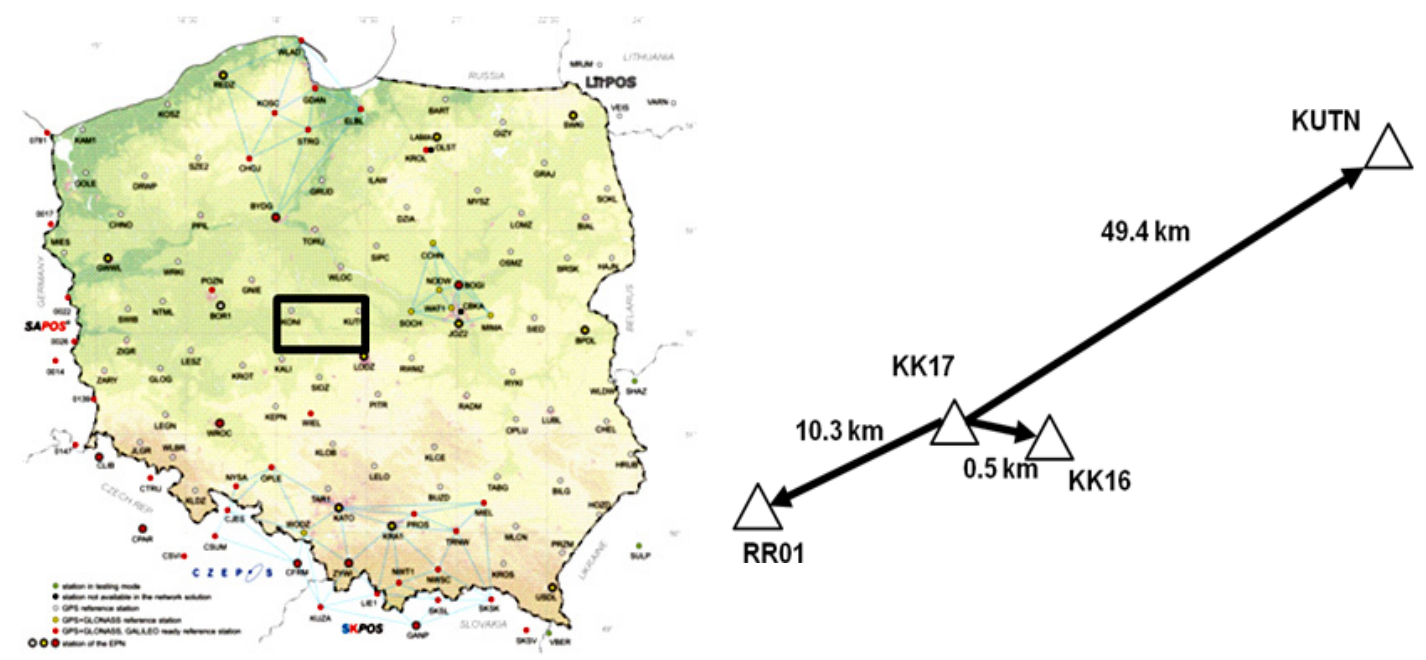

Fig. 1 The location of the test surveys.

http://www.asgeupos.pl/webpg/graph/dwnld/map_en_dwnld.jpg

\section{PROCEDURE 3 (AMBIGUITY-TEST)}

Third test is based on idea presented by Chen and $\mathrm{Wu}$ (Chen and $\mathrm{Wu}, 2013)$.

Matrix $\boldsymbol{Q}_{a z}(7)$ is decomposed:

$$
\boldsymbol{Q}_{a z}=\boldsymbol{G}^{\mathrm{T}} \boldsymbol{G}
$$

It was shown in (Chen and $\mathrm{Wu}, 2013)$ that fully decorrelated matrix can be presented as:

$\boldsymbol{Q}_{a \mathrm{zf}}=\boldsymbol{T} \boldsymbol{Q}_{a \mathrm{z}} \boldsymbol{T}^{\mathrm{T}}$,

with determinant preserving transformation matrix $\boldsymbol{T}$ :

$\boldsymbol{T}=\sqrt[\mathrm{n}]{\operatorname{det}(\boldsymbol{G})} \boldsymbol{G}^{-\mathrm{T}}$

where $\mathrm{n}$ is a number of ambiguities.

Hence, taking into account (20)-(22):

$\boldsymbol{Q}_{\mathrm{azf}}=\sqrt[\mathrm{n}]{\operatorname{det}(\boldsymbol{G})^{2}} \underset{\mathrm{n} \times \mathrm{n}}{\boldsymbol{I}}$

Diagonal matrix $\boldsymbol{Q}_{\text {azf }}$ preserves determinant of matrix $\boldsymbol{Q}_{\mathrm{az}}$ and has all elements equal.

An ambiguity vector of the float solution $\boldsymbol{a}_{0}$ and an ambiguity vector of the final solution $\boldsymbol{a}_{\mathrm{fix}}$ are transformed using $\boldsymbol{T}$ matrix:

$\boldsymbol{a}_{0 \mathrm{t}}=\boldsymbol{T} \boldsymbol{a}_{0}$

$\boldsymbol{a}_{\text {fixt }}=\boldsymbol{T} \boldsymbol{a}_{\text {fix }}$

where $\boldsymbol{a}_{0 \mathrm{t}}$ and $\boldsymbol{a}_{\mathrm{fixt}}$ are: transformed ambiguity vector of the float solution and transformed ambiguity vector of the final solution, respectively.

The difference:

$\boldsymbol{d a}=\boldsymbol{a}_{\mathrm{fixt}}-\boldsymbol{a}_{0 \mathrm{t}}$

is used for forming the statistic: $\mathrm{s}_{5}=\boldsymbol{d} \boldsymbol{a}^{T} \boldsymbol{Q}_{a \mathrm{zf}}^{-1} \boldsymbol{d a}=\frac{\boldsymbol{d a ^ { T } \boldsymbol { d a }}}{\sqrt[\mathrm{n}]{\operatorname{det}(\boldsymbol{G})^{2}}} \sim \chi_{\mathrm{n}}^{2}$

On the basis of formula:

$\mathrm{P}\left(\chi_{\mathrm{n}}^{2}>\gamma_{0}\right)=1-\mathrm{p}_{0}$

the critical value ${ }_{0}$ is determined. Hence the following criterion is assumed in ambiguity test:

If $\frac{s_{5}}{\gamma_{0}}>1$ then solution is rejected.

Otherwise it can be accepted.

\section{EXPERIMENT DESIGN}

The proposed approach was tested using the real GPS data of three baselines. The data come from campaign performed in order to monitor local deformation in open-pit mine "Adamów” in Central Poland (Baryła et al., 2011). Figure 2 depicts the location of the measurement area and the layout of baselines. One GPS station of ASG-EUPOS, Polish part of European Positioning System active geodetic network, was used in test surveys ("KUTN"). The surveys were performed on December 9th, 2008, on $49.4 \mathrm{~km}, 10.3 \mathrm{~km}$ and $0.5 \mathrm{~km}$ baselines, with a 30 second sampling rate. Data sets of each baseline consisted of 100 epochs. The data were processed independently for each epoch. The approximate position was computed using code-observations in DGPS mode basing on single reference station KK17. A carrier phase data set was processed according to the algorithm of the MAFA method. The "true" coordinates were derived using Bernese software based on an 8-hour data set. Validation was performed using three methods described in previous section. 


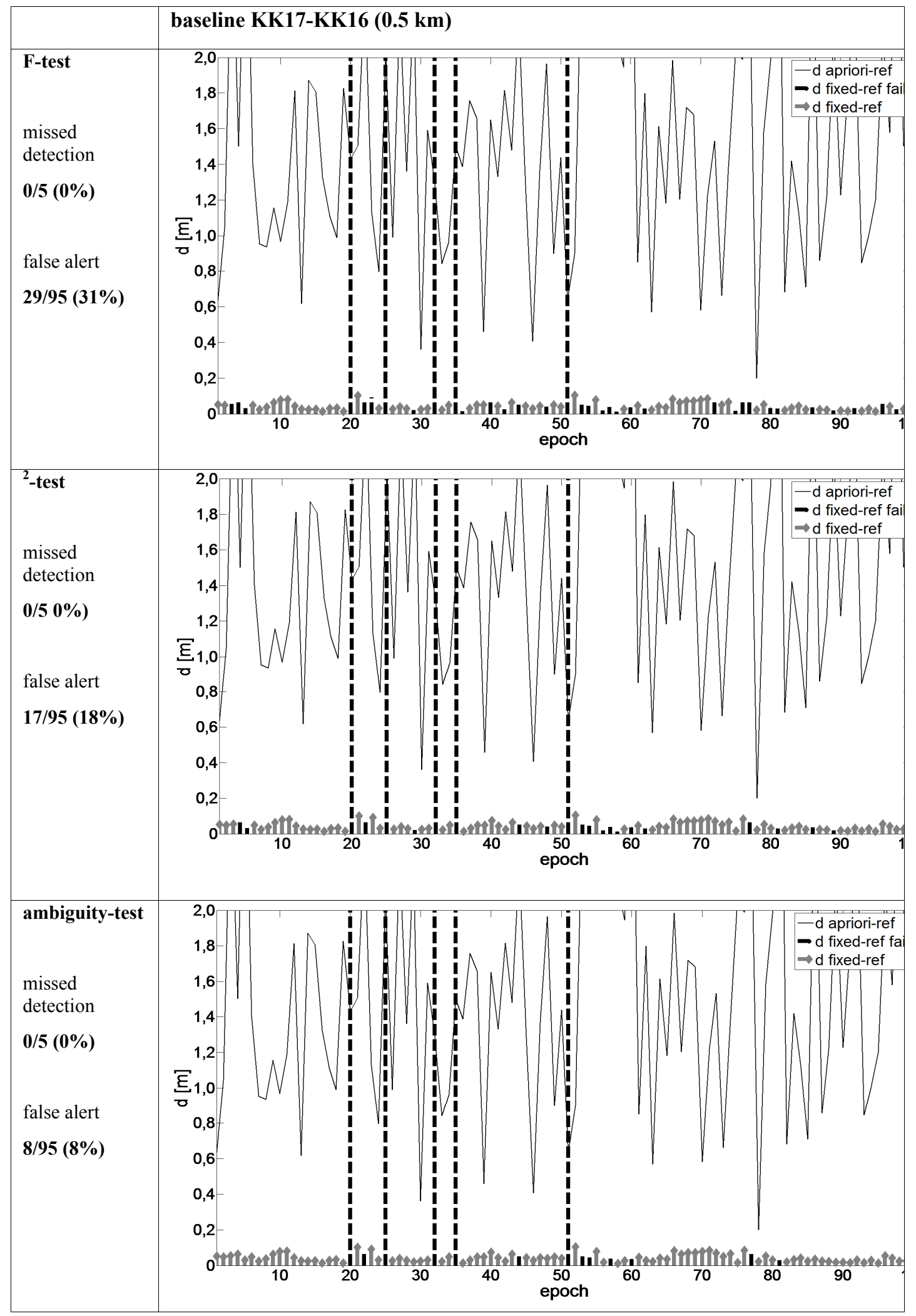

Fig. 2 Residuals of position referenced to the true position. Baseline $0.5 \mathrm{~km}$. 


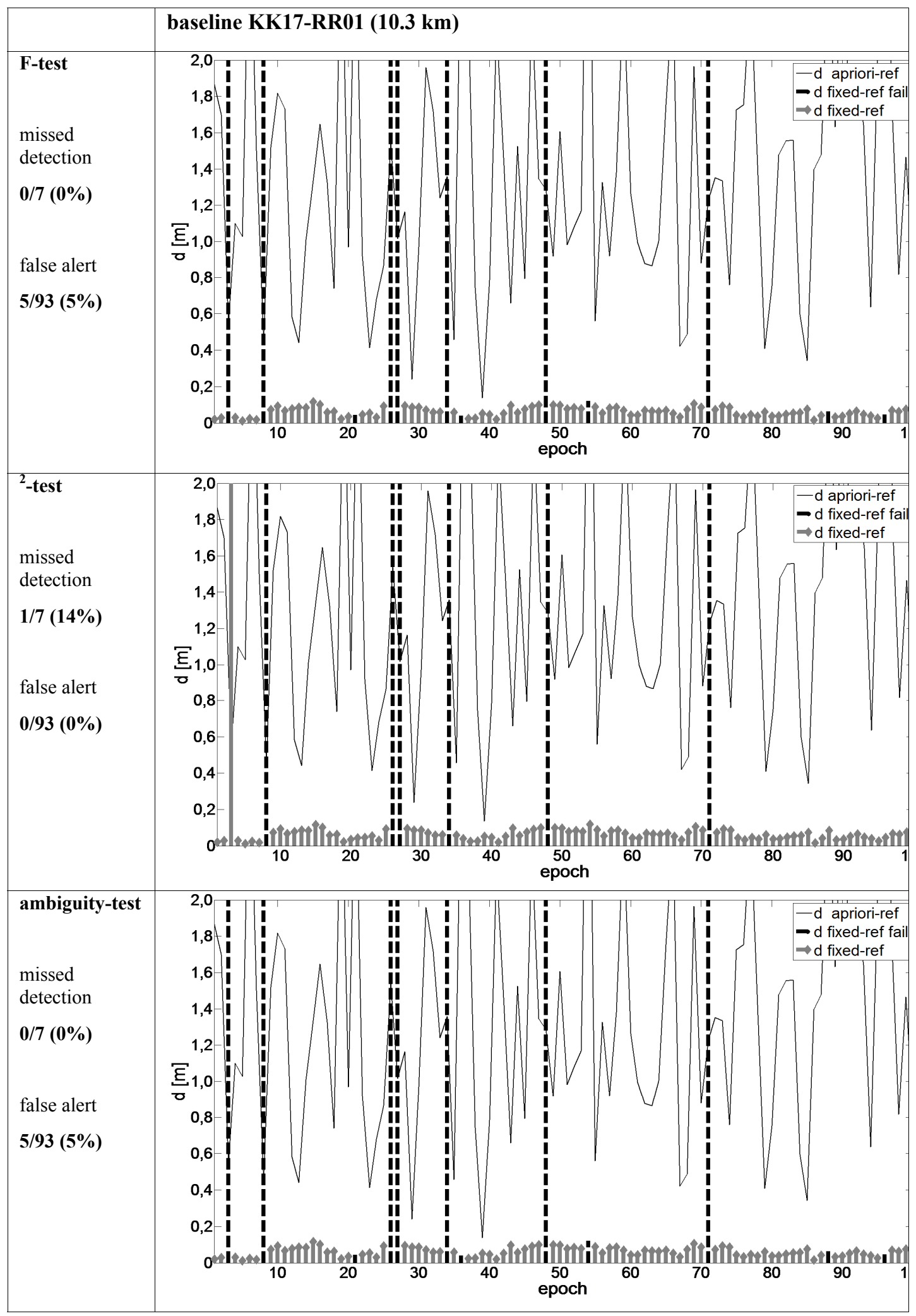

Fig. 3 Residuals of position referenced to the true position. Baseline $10.3 \mathrm{~km}$. 


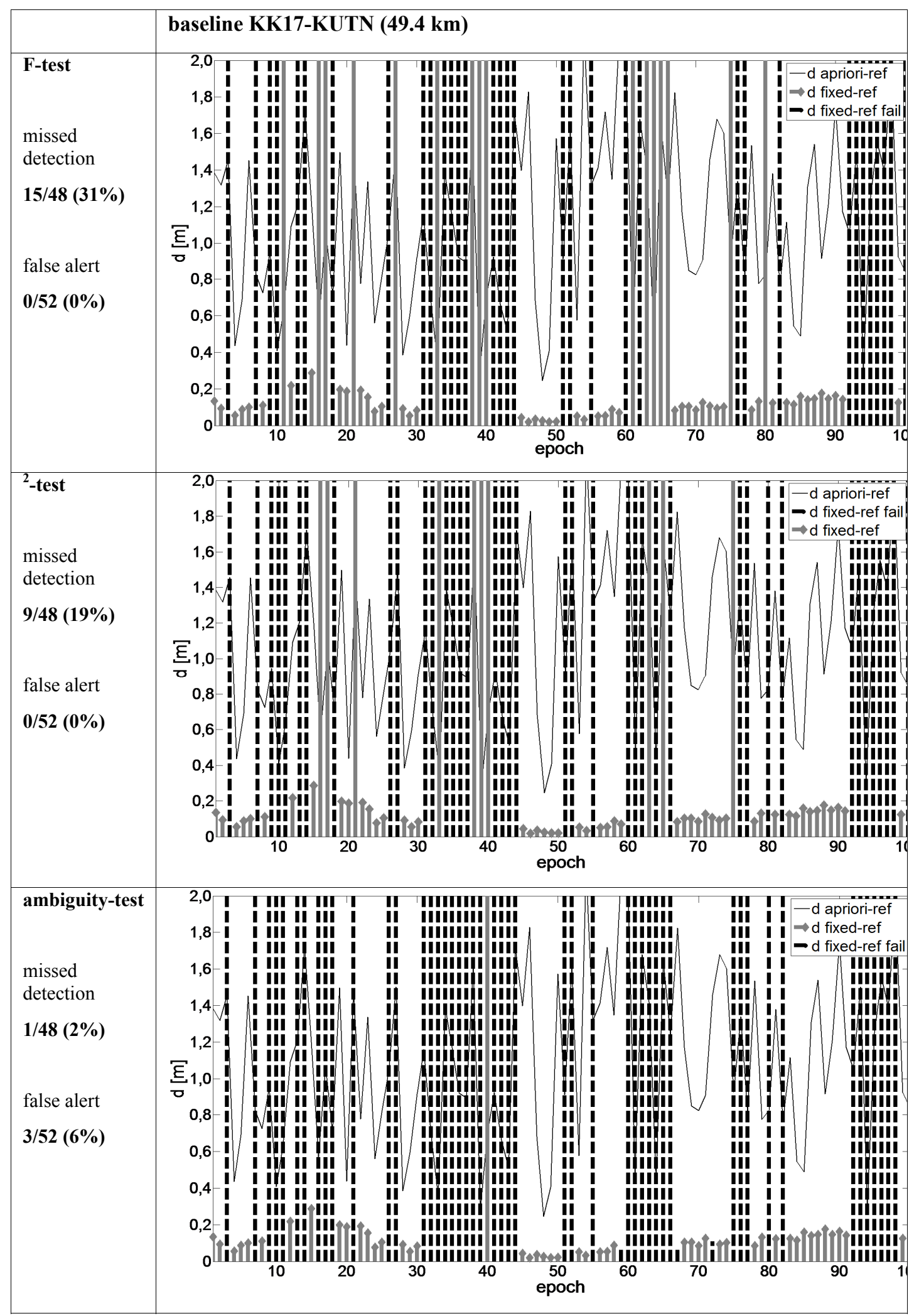

Fig. 4 Residuals of position referenced to the true position. Baseline $49.4 \mathrm{~km}$. 


\section{TESTS RESULTS}

Figures 2-4 depict 3D residuals of the position obtained from the single epoch positioning using MAFA method, with respect to reference position which was computed using Bernese software on the basis of 4 hour data set. The coordinate residuals were computed as: $\mathrm{d}=\sqrt{\Delta X^{2}+\Delta Y^{2}+\Delta Z^{2}}$, where $\Delta X, \Delta Y$ and $\Delta Z$ are components of the residuals with respect to reference position. The black, dashed lines depict the solutions that did not pass validation test, whereas grey lines with diamond marks depict solutions that pass validation test. The thin, solid line depicts the linear residuals of the a priori position (DGPS), with respect to the reference position. Figure 2 concerns $0.5 \mathrm{~km}$ baseline. In the case of the F-test, 5 among 100 solutions were wrong and they were correctly identified by the validation procedure. Unfortunately, also 29 correct solutions did not pass the test, which means that they were falsely rejected. In the $\chi^{2}$-test also 5 wrong solutions were correctly identified. However, in this case the number of correct solutions that did not pass the test equals 17 . The ambiguity test also has identified all wrong solutions correctly. In this case, the lowest amounts of correct solutions that did pass the test was obtained.

Figure 3 shows the results of $10.3 \mathrm{~km}$ baseline processing. There are 7 outliers. All of them were identified by F-test and ambiguity-test. In the case of the $\chi^{2}$-test one wrong solution was not identified. The ambiguity test and F-test point out to some solutions as incorrect, while actually they are correct. In the case of the $\chi^{2}$-test all correct solutions pass the test. Figure 4 depicts the results of $49.4 \mathrm{~km}$ baseline processing. In this case, 48 from 100 obtained solutions were incorrect. The number of incorrect solutions that was not identified by the validation procedure equals 15 in the case of the F-test, 9 in the case of the $\chi^{2}$-test and 1 in the case of ambiguity-test. This represents accordingly $31 \%, 19 \%$ and $2 \%$ of all incorrect solutions. All correct solutions passed the Ftest and the $\chi^{2}$-test, whereas the ambiguity-test points out to 3 correct solutions as incorrect.

Summarizing, number of wrong solutions that were identified by the test depends of the type of test and length of baseline. This value varies between $69 \%$ (F-test in the case of $49.4 \mathrm{~km}$ baseline) and $100 \%$ (all tests in the case of $0.5 \mathrm{~km}$ baseline and F-test as well as ambiguity-test for the case of $10.3 \mathrm{~km}$ baseline). Among three examined tests the best results were obtained using ambiguity-test. Only one incorrect solution was not identified in the case of baseline $49.4 \mathrm{~km}$. In the case of other baselines all incorrect solutions were identified.

As one can see, the results of these three methods differ slightly. No definite explanation of this effect is given in this paper. Nevertheless, one can make some guesses concerning this problem. The reason for the different results between the first and the second procedure can be that in the first procedure more information is utilized than in the second. These are: the weight matrix $\left(\mathbf{P}_{\mathrm{c}}\right)$, and residuals vector $\left(\boldsymbol{v}_{\mathrm{c}}\right)$ of the observations used for obtaining the float solution. The third procedure, unlike both previous procedures, relies on the confidence region formed in the ambiguity domain. The reason for the difference in the results could be that the confidence region formed in the ambiguity domain (in third procedure) and the region formed in the coordinates domain (in the first and the second procedures) are not equivalent. Nevertheless, those guesses are only hypotheses and require verification. This problem will be the subject of further research.

\section{CONCLUSIONS}

Most of incorrect solutions computed using MAFA method are far away from a priori position (float solution). This allows to develop validation techniques based on forming confidence region around float position and then testing if final solution fall into it or not. Three validation techniques were proposed in the paper. In the case of the first two techniques, the confidence region is determined in the coordinate domain whereas in the third technique it is formed in the ambiguity domain. All validation techniques were tested in single-epoch, precise, positioning mode. Three baselines with different length were processed. All of them gave good results. The percentage of identification incorrect solutions varied from $69 \%$ (F-test in $49.4 \mathrm{~km}$ baseline) to $100 \%$ in most of the rest cases. The best results gave ambiguity-test. This test identified all incorrect solutions in the cases of 0.5 and $10.3 \mathrm{~km}$ baselines and $98 \%$ incorrect solutions in the case of $49.4 \mathrm{~km}$ baseline.

\section{ACKNOWLEDGMENTS}

This research was supported by grant with agreement number UMO-2014/13/B/ST10/02547 from Polish National Center of Science.

\section{REFERENCES}

Abidin, H.: 1993, Computational and geometrical aspects of on-the-fly ambiguity resolution, Ph.D.Thesis, Dept. of Surveying Engineering, Tech. Report No. 104, University of New Brunswick, Canada.

Baryła, R., Wielgosz, P., Paziewski, J. and Błaszczyk, S.: 2011, Principles of ground deformation monitoring at open pit mine with use of GPS technology: KWB "Adamów" in Turek case study. Reports on Geodesy, No. 1 (90), 23-30.

Caspary, W. F., Haen, W. and Borutta, H.: 1990, Deformation analysis by statistical methods. Technometrics, 39(1), 49-57.

Caspary, W. F.: 2000, Concepts of network and deformation analysis. The University of New South Wales, Kensington, Australia.

Cellmer, S., Wielgosz, P. and Rzepecka, Z.: 2010, Modified ambiguity function approach for GPS carrier phase positioning. Journal of Geodesy, 84, 264-275. DOI: $10.1007 / \mathrm{s} 00190-009-0364-8$ 
Cellmer, S.: 2011a, The real time precise positioning using MAFA method. The $8^{\text {th }}$ International Conference Environmental Engineering, selected papers, III, Vilnius, 1310-1314.

Cellmer, S.: 2011b, Using the integer decorrelation procedure to increase of the efficiency of the MAFA Method. Artificial Satellites, 46, No. 3, 103-110. DOI: $10.2478 / \mathrm{v} 10018-012-0002-1$

Cellmer, S.: 2012a, A graphic representation of the necessary condition for the MAFA method. IEEE Transactions on Geoscience and Remote Sensing, $50(2), 482-88$.

Cellmer, S.: 2012b, On-the-fly ambiguity resolution using an estimator of the modified ambiguity covariance matrix for the GNSS positioning model based on phase data. Artificial Satellites, 47, No. 3, 81-90. DOI: 10.2478/v10018-012-0015-9

Cellmer, S.: 2012c, Single-epoch precise positioning using Modified Ambiguity Function Approach. Technical Sciences, 16(4), 265-280.

Cellmer, S.: 2013, Search procedure for improving Modified Ambiguity Function Approach. Survey Review, 45(332), 380-385.

DOI: $10.1179 / 1752270613 Y .0000000045$

Chen, Y.Q.: 1983, Analysis of deformation surveys- a generalized method. Technical Report No. 94, University of New Brunswick, Fredericton, 54-72.

Chen, Y.Z. and $\mathrm{Wu}, \mathrm{J} .:$ 2013, Zero-correlation transformation and threshold for efficient GNSS carrier phase ambiguity resolution. Journal of Geodesy, 87, 971-979.

DOI: $10.1007 / \mathrm{s} 00190-013-0661-0$

Euler, H. and Schaffrin, B.: 1991, On a measure for the discernibility between different ambiguity solutions in the static-kinematic GPS-mode. In: IAG Symposia No. 107, kinematic systems in geodesy, surveying, and remote sensing. Springer, New York, 285-295.

Euler, H. and Land, H.: 1992, Fast GPS ambiguity resolution on-the-fly for real-time application. In: Proceedings of Six International Geodetic Symposium on Satellite Positioning, Columbus, Ohio, 17-20 March, 650-659.

Frei, E. and Beutler, G.: 1990, Rapid static positioning based on the fast ambiguity resolution approach FARA: theory and first results. Manuscripta Geodaetica, 15(4), 325-356.

Han, S. and Rizos, C.: 1996, Improving the computational efficiency of the ambiguity function algorithm. Journal of Geodesy, 70, No. 6, 330-341.

DOI: $10.1007 / \mathrm{s} 001900050024$

Han, S.: 1997, Quality control issues relating to instantaneous ambiguity resolution for real-time GPS kinematic positioning. Journal of Geodesy, 71, 351361. DOI: $10.1007 / \mathrm{s} 001900050103$

Hassibi, A. and Boyd, S.: 1998, Integer parameter estimation in linearmodels with applications to GPS. IEEE Trans Signal Proc, 46, 2938-2952.

Hefty, J.: 2007, Geo-kinematics of Central and South-East Europe resulting from combination of various regional GPS velocity fields. Acta Geodyn. Geomater., 4, No. 4 (148), 173-189.

Hofmann-Wellenhof, B, Lichtenegger, H. and Wasle, E.: 2008, GNSS-Global Navigation Satellite Systems GPS, GLONASS, Galileo \& more, Springer-Verlag, Wien
Jung, J. and Enge, P.: 2000, Optimization of cascade integer resolution with three civil GPS frequencies. Proc. ION GPS'2000, Salt Lake City, September 2000.

Kontny, B., Bosy, J. and Borkowski, A.: 2006, The use of permanent and epoch GPS coordinate time series in geodynamic investigations of Sudetes area - proposal of a new approach. Acta Geodyn. Geomater., 3, No. 3 (143), 31-38.

Leick, A.: 2004, GPS Satellite Surveying. $3^{\text {rd }}$ edition, John Wiley and Sons, Inc.

Li, T. and Wang, J.: 2012, Some remarks on GNSS integer ambiguity validation methods. Survey Review, 44 (326), 230-238. DOI: $10.1179 / 17.52270611$ Y.0000000027

Lidberg, M., Johansson, J., Scherbeck, H.-G. and Milne, G.: 2010, Recent results based on continuous GPS observations of the GIA process in Fennoscandia from BIFROST. Journal of Geodynamics, 50, No. 1, 8-18. DOI: 10.1016/j.jog.2009.11.010

Liu, L.T., Hsu, H.T., Zhu, Y.Z. and Ou, J.K.: 1999, A new approach to GPS ambiguity decorrelation. Journal of Geodesy, 73, 478-490. DOI: 10.1007/PL00004003

Nowel, K.: 2015a, Robust M-estimation in analysis of control network deformations: Classical and new method. Journal of Surveying Engineering. DOI: 10.1061/(ASCE)SU.1943-5428.0000144

Nowel, K.: 2015b, Application of Monte Carlo method to statistical testing in deformation analysis based on robust Mestimation. Survey Review. DOI: 10.1179/1752270615Y.0000000026

Schenk, V., Schenkova, Z., Bosy, J. and Kontny, B.: 2010, Reliability of GPS data for geodynamic studies. Case study: Sudeten area, The Bohemian Massif. Acta Geodyn. Geomater., 7, No 1 (157), 113-128.

Teunissen, P.J.G.: 1995, The least-squares ambiguity decorrelation adjustment: a method for fast GPS integer ambiguity estimation. Journal of Geodesy, 70, 65-82. DOI: 10.1007/BF00864319

Teunissen, P.J.G.: 1998a, On the integer normal distributions of the GPS ambiguities. Artificial Satellites, 33, No. 2, 49-64.

Teunissen, PJG. and Kleusberg, A.: 1998b, GPS for Geodesy, Springer - Verlag, Berlin Heidelberg New York.

Teunissen, P.J.G.: 1999, An optimality property of the integer least-squares estimator. Journal of Geodesy, 73, No. 11, 587-593. DOI: 10.1007/s00190050269

Teunissen, P.J.G.: 2002, The parameter distributions of the integer GPS model. J Geodesy, 76, No. 1, 41-48. DOI:10.1007/s00190010023

Teunissen, P.J.G.: 2003, Integer aperture GNSS ambiguity resolution. Artificial Satellites, 38(3), 79-88.

Teunissen, P.J.G.: 2004, Penalized GNSS ambiguity resolution. Journal of Geodesy 78, 235-244. DOI: $10.1007 / \mathrm{s} 00190-004-0393-2$

Teunissen, P.J.G.: 2005, A carrier phase ambiguity estimator with easy-to evaluate fail-rate. Artificial Satellites, 38(3), 89-96.

Teunissen P.J.G. and Verhagen, S.: 2009, The GNSS ambiguity ratio-test revisited: a better way of using it. Survey Review, 41(312), 138-151. DOI: $10.1179 / 003962609 X 390058$

Teunissen, P.J.G.: 2013, GNSS integer ambiguity validation: Overview of theory and methods. Proceedings of the ION 2013 Pacific PNT Meeting, Honolulu, Hawaii, April 2013, 673-684. 
Tiberius, C. and Jonge, P.: 1995, Fast positioning using the LAMBDA method. In: Proceedings of DSNS-95, Bergen, Norway, Paper No. 30.

Urquhart, L.: 2009, An analysis of multi-frequency carrier phase. Linear combinations for GNSS. Senior Technical Report No. 263. Department of Geodesy and Geomatics Engineering, University of New Brunswick. Fredericton, New Brunswick, Canada, $71 \mathrm{pp}$.

Verhagen, S.: 2004. Integer ambiguity validation: An open problem? GPS Solutions, 8(1), 36-43.

DOI: $10.1007 / \mathrm{s} 10291-004-0087-5$

Verhagen, S.: 2005, The GNSS integer ambiguities: estimation and validation. Ph.d. thesis, Publications on Geodesy, 58, Netherlands Geodetic Commmission, Delft.

Wielgosz, P., Cellmer, S., Rzepecka, Z. Paziewski, J. and Grejner-Brzezinska, D.A.: 2011, Troposphere modeling for precise GPS rapid static positioning in mountainous areas. Measurement Science and Technology, 22, No. 4, 89-99.

DOI: $10.1088 / 0957-0233 / 22 / 4 / 045101$

Wang, J., Stewart, M. and Tsakiri, M.: 1998, A discrimination test procedure for ambiguity resolution on-the-fly. Journal of Geodesy, 72, 644-653. DOI: $10.1007 / \mathrm{s} 001900050204$

Wang, J., Stewart, M and Tsakiri, M.: 2000, A comparative study of the integer ambiguity validation procedures. Earth Planets Space, 52, No. 10, 813-817.

Xu, P.: 2001, Random simulation and GPS decorrelation. Journal of Geodesy 75, 408-423. DOI: $10.1007 / \mathrm{s} 001900100192$

$\mathrm{Xu}$, P.: 2006, Voronoi cells, probabilistic bounds, and hypothesis testing in mixed integer linear models. IEEE Transactions on Information Theory, 52, 31223138. 